\section{OWE-27 ANALYSIS OF UC COLECTOMY RATES IN PRE- AND POST- BIOLOGIC ERA SOUTH-EAST SCOTLAND}

${ }^{1}$ Philip Jenkinson*, 'Gareth Jones, 'Nik Plevris, 'Mathew Lyons, "Kate Kirkwood, ${ }^{1}$ David Wilson, ${ }^{1}$ Malcolm Dunlop, ${ }^{1}$ Ian Arnott, ${ }^{1}$ Charlie Lees. ${ }^{1}$ Nhs Lothian, Edinburgh, UK; ${ }^{2}$ University of Edinburgh, Edinburgh, UK

\subsection{6/gutjnl-2019-BSGAbstracts.360}

Introduction The use of biologic therapy for UC has lagged significantly behind that of Crohn's Disease in part because of restricted guidance from NICE. Following approvals of antiTNF and vedolizumab for UC in early 2015 a significant increase in prescribing has been reported. Whether this has resulted in a decrease in colectomy rates has not been studied in a real-world setting. The aim of this study was to describe trends in colectomy rates for UC over time spanning the preand post-biologic era.

Methods All patients (adult and paediatric) with a diagnosis of UC who received maintenance biologic treatment and/or underwent a colectomy in Lothian, Scotland between 1st January 2005 and 31st December 2018 were identified by interrogating multiple clinical and administrative databases. Patient phenotype, prescribing and surgical data including complications were extracted by manual review of the electronic medical record. Linear and segmental regression were used to identify changes in the trend of biologic prescription and colectomy rates and to estimate the annual percent change. Colectomy rates were described per 100 UC patients, with yearly prevalence data obtained from the Lothian IBD Registry.

Results Of 175 patients treated with maintenance biologic therapy 14 (8\%) were initiated 2005 to 2013 and 161 (92\%) from 2014 to 2018 (Figure 1A). The number of patients starting treatment per year increased from 0.06 in 2005 to 1.37 in 2018 per 100 UC patients $(p<0.001)$. A total of 448 patients underwent colectomy. Colectomy rates per $100 \mathrm{UC}$ patients fell throughout the study period; from 1.84 in 2005 to 0.45 in $2018(\mathrm{p}<0.001)$. On segmental regression a joinpoint was identified at 2014; the annual colectomy rate fell by $5.81 \%$ per year from 2005 to 2014 and by $17.41 \%$ per colectomy following biologic treatment (median duration 7.5 months), there was no increase in post-operative complication rates in biologic exposed patients vs no biologics.

Conclusion The increase in biologic prescribing for UC has been parallelled by a significant reduction in colectomy rates without increased post-operative complications. Whilst we cannot ascribe causality further work is under way to expand this cohort across Scotland. year from 2014 to 2018 ( $\mathrm{p}<0.05) .41 / 175$ patients underwent

\section{OWE-28 POST-IMAGING COLORECTAL CANCER IN THE ENGLISH NATIONAL HEALTH SERVICE BOWEL CANCER SCREENING PROGRAMME}

${ }^{1}$ Edmund Derbyshire*, ${ }^{2}$ Andrew Plumb, ${ }^{3}$ Claire Nickerson, ${ }^{3}$ Billie Moores, ${ }^{4}$ Suzanne Wright, ${ }^{5}$ Eva Morris, ${ }^{6}$ Roland Valori, ${ }^{7}$ Matt Rutter. ${ }^{1}$ Royal Liverpool and Broadgreen University Hospitals Nhs Trust, Liverpool, UK; ${ }^{2}$ University College Hospitals NHS Foundation Trust, London, UK; ${ }^{3}$ Public Health England, Manchester, UK; ${ }^{4}$ Public Health England, Sheffield, UK; ${ }^{5}$ University of Leeds, Leeds, UK; ${ }^{6}$ Gloucestershire Hospitals NHS Foundation Trust, London, UK; ${ }^{7}$ North Tees and Hartlepool NHS Foundation Trust, Stockton-on-Tees, UK

\subsection{6/gutjnl-2019-BSGAbstracts.361}

Introduction Computed tomography colonography (CTC) is the gold standard radiological investigation for colorectal cancer screening. The Post-Imaging Colorectal Cancer (PICRC) rate (i.e. colorectal cancers that develop after a negative CTC) is a key quality indicator of CTC; however, PICRC rates have not been previously reported in a national programme.

The Word Endoscopy Organisation has, through a consensus process, agreed a standard methodology for calculating PICRC rates to enable international benchmarking (1). This rate (which the WEO terms PICRC-3y) is calculated by dividing the PICRCs detected 6 months to 3 years after a negative CTC (false negatives) by the sum of the true positives (defined by CTCs with a cancer diagnosis within 6 months) and false negatives (PICRCs). This study aimed to determine the rate of PICRC-3y in the English National Health Service (NHS) Bowel Cancer Screening Programme (BCSP).

Methods Data from each Bowel Cancer Screening Programme (BCSP) CTC is entered into a national database, the Bowel Cancer Screening System (BCSS). All colorectal adenocarcinomas, within and outside the BCSP, are validated and registered by the National Cancer Registration and Analysis Service (NCRAS). This retrospective observational study interrogated these databases to identify BCSP true positive and false negative CTCs. CTCs were included regardless of whether they were preceded or followed by a colonoscopy within a screening episode.

Results

\begin{tabular}{|c|c|c|c|c|}
\hline & $\begin{array}{l}\text { True Positive } \\
\text { СTC }\end{array}$ & $\begin{array}{l}\text { False Negative } \\
\text { CTC }\end{array}$ & $\begin{array}{l}\text { To year } \\
\text { end }\end{array}$ & PICRC-3y Rate \\
\hline $\begin{array}{l}200- \\
008\end{array}$ & 18 & 1 & 200-011 & $5.26 \%(95 \% \mathrm{Cl} 0 .-6)$ \\
\hline 2009 & 45 & 2 & 2012 & $\begin{array}{l}4.26 \%(95 \% \mathrm{Cl} 0 .- \\
4.5)\end{array}$ \\
\hline 2010 & 55 & 5 & 2013 & $\begin{array}{l}\text { 8.33\% }(95 \% \mathrm{Cl} 3 .- \\
8.1)\end{array}$ \\
\hline Total & 118 & 8 & & $\begin{array}{l}6.35 \%(95 \% \text { Cl 2.- } \\
2.1)\end{array}$ \\
\hline
\end{tabular}
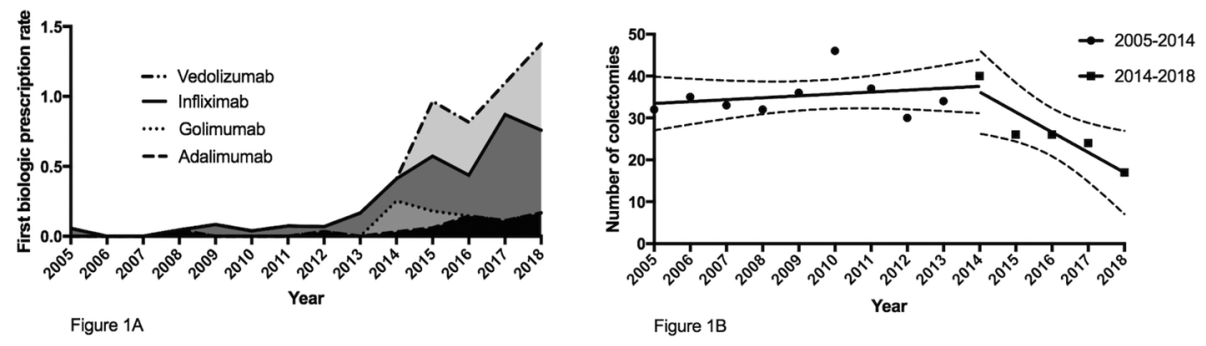

Abstract OWE-27 Figure 1 
Of the 8 PICRCs, 3 were detected at subsequent BCSP procedures, one at 1 year surveillance, and 2 following re-invitation for screening at 2 years. Five were detected outside the BCSP. Conclusions

1. The PICRC-3y in the BCSP is higher than the rate in the published literature (4.4\%)(2) and the corresponding rate for Post Colonoscopy Colorectal Cancer (PCCRC) over the same period $(2.5 \%)$.

2. CTC is commonly reserved for patients who are either deemed unsuitable for colonoscopy, or in whom colonoscopy has failed, meaning that there are likely to be substantial differences between the populations undergoing each examination.

3. Because CTC and colonoscopy are performed in different populations, those having CTC more likely to be frailer with greater co-morbidity, the higher rate of PICRC when compared to PCCRC has to be interpreted with caution and may relate to the difficulty of investigating such patients, who may not be fit enough for, or refuse an intervention even if a lesion is found.

\section{REFERENCES}

1. Beintaris I, et al. United European Gastroenterology Journal Vol 5 Issue 5_Suppl P0436

2. Obaro AE, et al. Lancet Gastroenterol Hepatol 3(5) 32-36

\section{Abstracts of Distinction}

\section{AWE-08 WHY ARE WE MISSING COLORECTAL CANCER? A STUDY INVESTIGATING THE CAUSE OF DELAYS IN DIAGNOSIS}

B Hayes, I Bjarnason, R Logan, B Hayee, P Dubois, A Haji, Lindsey Barker, G Chung-Faye, R Srirajaskanthan, AJ Kent. King's College Hospital, London, UK

\subsection{6/gutjnl-2019-BSGAbstracts.362}

Introduction Delay in diagnosis of colorectal cancer (CRC) is associated with worse outcomes. Although studies have shown the incidence of CRC missed at endoscopy to be $2.5 \%-7.7 \%$ (Morris et al., 2014), there are additional non-endoscopic factors that may lead to delays. This study aimed to identify all factors leading to a delayed diagnosis, including endoscopic "misses".

Methods All patients diagnosed with CRC at Kings College Hospital, London between 2011 - 2018 were included. We identified patients seen in an outpatient clinic or underwent endoscopy within 36 months preceding diagnosis. 'Delayed' cancers were grouped into 'clinical factors' and 'technical factors'. 'Clinical factors' included the subset of post-colonoscopy colorectal cancers (PCCRC). The Joint Advisory Group on GI endoscopy (JAG) have defined PCCRC as being cancers diagnosed within 36 months of an endoscopy.

Results 797 cases of CRC were diagnosed in the study period and $60(7.5 \%)$ were seen in the preceding 36 months. 46 patients $(5.8 \%)$ were determined to have a delayed diagnosis, of which $24(52.2 \%)$ were diagnosed within 1 year of initial investigation. 38 delayed diagnoses were due to clinical factors: PCCRC $(n=23)$, incomplete endoscopy $(n=2)$, inadequate investigation $(n=7)$, missed on rigid sigmoidoscopy $(\mathrm{n}=1)$ and incomplete bowel preparation $(\mathrm{n}=5)$ with an average delay of 172 days. 8 delays were caused by technical factors: Incomplete follow up $(\mathrm{n}=4)$, delayed investigation $(n=2)$, histology not reviewed $(n=1)$, missed on CT $(n=1)$.

The rate of missed cancer in the $2 \mathrm{WW}$, Bowel Cancer Screening and Routine referral pathways was 4.8\% ( $n=14)$, $5.1 \% \quad(n=8), \quad 7.3 \% \quad(n=28) \quad$ respectively. The incidence of missed cancer in the right colon was significantly higher $(p=0.068,95 \%$ CI $0.9-.57)$. Further interrogation showed the highest incidence in the hepatic flexure $(10.5 \%)$, splenic flexure $(9.4 \%)$, caecum $(7.5 \%)$, and anal canal $(6.5 \%)$.

91.3\% of PCCRCs versus $47.8 \%$ of other missed cancers identified another pathology at the initial endoscopy which was documented on the report $(p=0.003,95 \%$ Confidence interval 2.1-0.6).

A delay in diagnosis was not associated with more advanced TNM staging or K-ras mutations.

Conclusions Our rate of missed cancers is equivalent to that published in the literature, with the majority of missed cancers due to PCCRC. Delays in diagnosis are related to avoidable factors, such as improving the quality of bowel prep or ensuring further investigations in patients with incomplete endoscopies. Endoscopists should also be aware of the increased miss rate in specific locations. Non-cancer pathology identified at endoscopy is also associated with missed cancer, so endoscopists should be careful to maintain a careful examination for concomitant cancers.

\section{Posters}

\section{PWE-039 INDICATORS OF RECURRENCE IN COMPLEX COLORECTAL POLYPS AT RESECTION}

${ }^{1}$ Ejaz Hossain*, ${ }^{2}$ Sreedhari Thayalasekaran, ${ }^{3}$ Asma Alkandari, ${ }^{4}$ Sophie Arndtz, ${ }^{5}$ Mohamed Abdelrahim, ${ }^{6}$ Pradeep Bhandari. 'Queen Alexandra Hospital, Portsmouth, UK; ${ }^{2} Q A$ Hospital, Portsmouth; ${ }^{3} Q A$ Hospital, Portsmouth; ${ }^{4} Q A$ Hospital, Portsmouth; ${ }^{5} Q A$ Hospital, Portsmouth; ${ }^{6}$ QA Hospital, Portsmouth

\subsection{6/gutjnl-2019-BSGAbstracts.363}

Cancer incidence in $-0 \mathrm{~mm}$ endoscopically detected colorectal lesions

Introduction Recent data has shown the risk of cancer in colorectal lesions $<5 \mathrm{~mm}$ is less than previously reported. This has lead to a 'resect and discard' strategy for diminutive polyps. Few studies have been done to demonstrate cancer incidence in lesions between $-0 \mathrm{~mm}$ and formulating a treatment strategy for these lesions.

Methods We analysed outcomes of all patients who underwent colonoscopy from January 2007 to December 2018 and were found to have colorectal polyps. Data was prospectively collected on an online endoscopy reporting system and pathology reporting system. A chart review was then carried out analysing the site, size, morphology and histological diagnoses of each polyp.

Results A total of 15906 polyps were removed at colonoscopy over the specified period, size of the polyps ranging from 1 $\mathrm{mm}$ to $120 \mathrm{~mm}$ and a mean size of $7.3 \mathrm{~mm}$.

A histopathological diagnosis of 104 cancers was made ( $0.65 \%$ of all polyps), of which 94 cancers $(90.25 \%)$ were associated with non pedunculated polyps [OR 1.45, 95\% CI 0.7-.78].

89 cancers were found in the left colon and rectum compared with 15 cancers in the right colon ( $85.5 \%$ vs $14.5 \%$ ) 